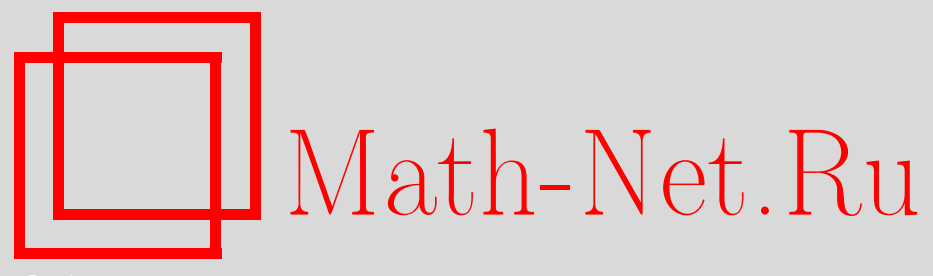

А. Нестеренко, Нетранзитивные кости, Квант, 2021, номер 10, 30-31

DOI: https://doi.org/10.4213/kvant20211004

Использование Общероссийского математического портала Math-Net.Ru подразумевает, что вы прочитали и согласны с пользовательским соглашением http://www.mathnet.ru/rus/agreement

Параметры загрузки:

IP : 52.6 .47 .48

26 апреля 2023 г., 14:09:35

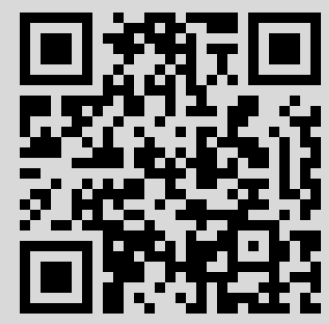




\section{Нетранзитивные КОСТИ}

\section{A. HECTEPEHKO}

\section{Нетранзитивный парадокс}

При игре в кости по обычным правилам выигрывает игральная кость, на которой выпала грань с большим числом. Если на двух костях разные наборы чисел, одна из них может оказаться сильнее другой в том смысле, что вероятность ее выигрыша больше 1/2 .

Возьмем такие три кости, что в каждой паре есть сильнейшая. Кажется, что среди этих трех костей должна найтись самая сильная, которая бьет две другие. Ведь если кость $A$ сильнее $B$, а кость $B$ сильнее $C$, здравый смысл подсказывает, что $A$ тем более сильнее $C$.

Это не так. На рисунке 1 показаны развертки трех костей с вероятностями выигрыша первой у второй $p_{1}=5 / 9$, второй у третьей $p_{2}=2 / 3$ и третьей у первой $p_{3}=2 / 3$. Такие кости называют нетранзитивными: из того, что $A$ сильнее $B$ и $B$ сильнее $C$, не следует, что $A$ сильнее $C$.

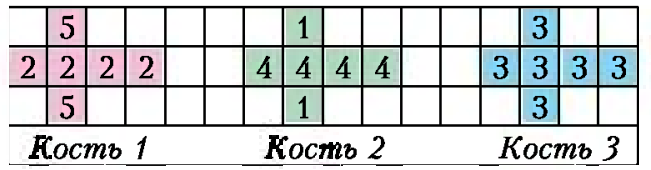

Рис. 1

Известна игра с нетранзитивными костими по таким правилам: играют двое, сначала они по очереди выбирают себе по кости, а дальше как обычно - выигрывает тот игрок, на чьей кости выпадает большее число. Кажется парадоксом, что тот, кто выбирает вторым, всегда может обеспечить себе вероятность выигрыша больше $\frac{1}{\underline{2}}$. Но это так. Первый игрок не может выбрать сильнейшую кость: в нетранзитивном наборе такой

Автор этой статьи Александра Нестеренко ученица 9 класса школы 1287 г. Москвы

DOI: https://doi.org/10.4213/kvant2021 1004 просто нет. Зато второй всегда может взять кость сильнее кости первого.

\section{Нетранзитивный волчок}

Чтобы разобраться, как работает такая нетранзитивность, и примирить здравый смысл с кажущимся парадоксом, рассмотрим игру в нетранзитивный волчок (рис.2).

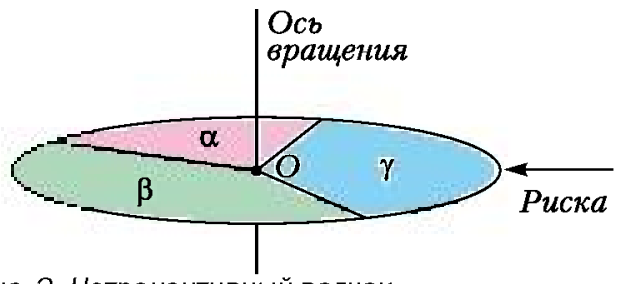

Рис. 2. Нетранзитивный волчок

Диск волчка, который крутится вокруг неподвижной вертикальной оси, разделен на три сектора с углами $\alpha, \beta$ и $\gamma$. Рядом с диском установлена неподвижная риска. Два игрока выбирают себе по сектору. Волчок раскручивают и смотрят, в каком положении он остановится. Сектор $\alpha$ в игре с $\beta$ проигрывает, если $\alpha$ окажется напротив риски (в остальных случаях $\alpha$ выигрывает). Сектор $\beta$ проигрывает в игре с $\gamma$, если напротив риски окажется сектор $\beta$. И $\gamma$ проигрывает в игре с $\alpha$, если напротив риски будет $\gamma$.

Будем измерять углы в долях полного оборота, так что $\alpha+\beta+\gamma=1$. Тогда вероятность проигрыша сектора $\alpha$ сектору $\beta$ равна $\alpha$, сектора $\beta$ сектору $\gamma$ равна $\beta$, и сектора $\gamma$ сектору $\alpha$ равна $\gamma$. Обозначим через $p_{1}, p_{2}$ и $p_{3}$, соответственно, вероятности выигрыша сектора $\alpha$ в игре с сектором $\beta$, сектора $\beta$ в игре с сектором $\gamma$ и сектора $\gamma$ в игре с сектором $\alpha$. Тогда $p_{1}=1-\alpha, p_{2}=1-\beta, p_{3}=1-\gamma$.

При $\alpha, \beta, \gamma<\frac{1}{2}$ каждая из вероятностей $p_{1}, p_{2}$ и $p_{3}$ больше $1 / 2$, т.е. игра нетранзитивная. Например, если секторы одинаковые

$$
(\alpha=\beta=\gamma=1 / 3) \text {, то } p_{1}=p_{2}=p_{3}=\frac{2}{3}>\frac{1}{2} \text {. }
$$

В игре с волчком сумма вероятностей выигрышей всегда равна 2:

$$
\begin{aligned}
p_{1}+p_{2}+p_{3}=1-\alpha+1-\beta+1-\gamma= \\
=3-(\alpha+\beta+\gamma)=2 .
\end{aligned}
$$

Предположим, что игрок, который выбирает сектор первым, делает это случайным об- 
разом, а тот, кто выбирает вторым, играет наилучшим образом. Тогда вероятность выигрыша второго равна $\frac{p_{1}+p_{2}+p_{3}}{. ;}-\frac{2}{;}$

\section{Нетранзитивные кости}

Потренировавшись с волчком, можно приступить к нетранзитивным костям.

Будем рассматривать наборы из трех шестигранных костей. Вероятности выигрыша первой кости у второй, второй у третьей и третьей у первой равны $p_{1}, p_{2}$ и $p_{3}$ соответственно. Для исключения ничьих примем, что на гранях разных костей не может быть одинаковых чисел. Более того, можно считать, что все 18 чисел различны, а именно, что каждое число от 1 до 18 встречается ровно один раз. Для простоты рассуждений ограничимся именно такими наборами.

Нам понадобится упрощенно представлять набор костей последовательностью их номеров. Построим такую последовательность на примере следующего набора костей (рис. 3) Разрежем развертки на отдельные грани и расположим эти грани в строке в порядке убывания чисел на них (рис. 4). Теперь для вычисления вероятностей выигрыша числа на гранях не нужны, ведь каждая грань бьет любую грань другой кости, стоящую правее. Поэтому числа на гранях заменим номерами костей, на которых эти грани находятся. Получается представление набора костей последовательностью номеров этих костей (рис. 5).

Такого представления достаточно для определения вероятностей $p_{1}, p_{2}$ и $p_{3}$ Например, чтобы найти $p_{1}$, надо для каждой единицы в последовательности найти количество двоек, стоящих правее этой единицы, сложить все эти числа и разделить на произведение количеств единиц и двоек:

$$
\begin{gathered}
p_{1}=\frac{4 \cdot \frac{6}{6 \cdot 6}}{=}=\frac{24}{36}=\frac{2}{3}, p_{2}=\frac{6 \cdot 4}{6 \cdot 6}=\frac{24}{36}=\frac{2}{3}, \\
p_{3}-\frac{2 \cdot 6+4 \cdot 2}{6 \cdot 6}-\frac{20}{36}-\frac{5}{9} .
\end{gathered}
$$

По представлению с вероятностями $p_{1}$, $p_{2}$ и $p_{3}$ всегда можно восстановить набор костей с теми же вероя̆тностйми (единственный с точностью до перестановок граней на каждой отдельной кости). Длй этого достаточно пронумеровать числа представления

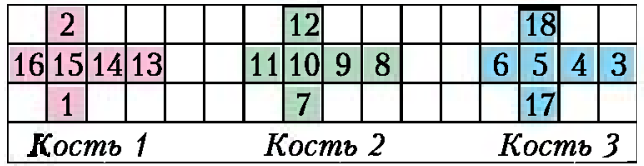

Рис. 3. Развертки трех костей

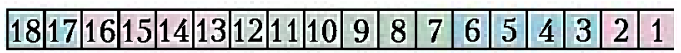

PHC. 4

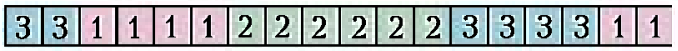

Рис. 5. Пример представления набора костей

$|3| 3|1| 1|1| 1|2| 2|2| 2|2| 2|3| 3|3| 3|1| 1$

\begin{tabular}{|l|l|l|l|l|l|l|l|l|l|l|l|l|l|l|l|l|l|}
\hline 3 & 3 & 1 & 1 & 1 & 2 & 2 & 1 & 2 & 2 & 2 & 2 & 2 & 3 & 3 & 3 & 3 & 1 \\
\hline
\end{tabular}

PHC. 6

слева направо в порядке убывания и нанести номера единиц на грани первой кости, номера двоек на грани второй и номера троек на грани третьей.

Если в представлении поменять местами соседние неравные номера, то одна из вероятностей $p_{1}, p_{2}$ и $p_{3}$ изменится на 1/36. Например, если в представлении, изображенном на рисунке 5 , единица «перепрыгнет» двойку слева направо (рис.6), получатся новый набор костей и новая последовательность, в которой количество фрагментов [1|2] уменьшится на 1 и, значит, вероятность $p_{1}$ уменьшится на 1/36 и станет равна 24/36-1/36=23/36. Вероятности $p_{2}$ и $p_{3}$ при этом не изменятся.

В таблице 1 приведены результаты всех возможных перестановок соседних номеров.

\begin{tabular}{|c|c|c|}
\hline Перестановка & \multicolumn{2}{|c}{ Результат } \\
\hline$[1 \mid 2] \rightarrow[2 \mid 1]$ & $p_{1} \rightarrow p_{1}-\frac{1}{36}$ & $p_{2}$ и $p_{3}$ не меняются \\
\hline$[1 \mid 3] \rightarrow[3 \mid 1]$ & $p_{3} \rightarrow p_{3}+\frac{1}{36}$ & $p_{1}$ и $p_{2}$ не меняются \\
\hline$[2 \mid 3] \rightarrow[3 \mid 2]$ & $p_{2} \rightarrow p_{2}-\frac{1}{36}$ & $p_{3}$ и $p_{1}$ не меняются \\
\hline$[2 \mid 1] \rightarrow[1 \mid 2]$ & $p_{1} \rightarrow \bar{p}_{1}+\frac{1}{36}$ & $p_{2}$ и $p_{3}$ не меняются \\
\hline$[3 \mid 1] \rightarrow[1 \mid 3]$ & $p_{3} \rightarrow p_{3}-\frac{1}{36}$ & $p_{1}$ и $p_{2}$ не меняются \\
\hline$[3 \mid 2] \rightarrow[2 \mid 3]$ & $p_{2} \rightarrow p_{2}+\frac{1}{36}$ & $p_{3}$ и $p_{1}$ не меняются \\
\hline
\end{tabular}

Табл. 1 


\section{\begin{tabular}{|l|l|l|l|l|l|l|l|l|l|l|l|l|l|l|l|l|}
\hline 1 & 1 & 1 & 1 & 1 & 1 & 2 & 2 & 2 & 2 & 2 & 2 & 3 & 3 & 3 & 3 & 3 \\
\hline
\end{tabular}}

Рис. 7. Представление из трех групп цифр

Если между двумя единицами в представлении двоек больше, чем троек, то правую единицу можно перенести вплотную к левой. При этом $p_{1}$ увеличится сильнее, чем уменьшится $p_{3}$, и сумма вероятностей возрастет. Если между двумя единицами в представлении троек больше, чем двоек, то левую единицу можно перенести вплотную к правой. И снова сумма вероятностей возрастет, так как $p_{3}$ увеличится сильнее, чем уменьшится $p_{1}$. Если между двумя единицами двоек и троек поровну, то любую из этих единиц можно перенести вплотную к другой без изменения суммы вероятностей. Значит, в любом представлении все единицы можно собрать в одну группу единиц, стоящих подряд, не уменьшив при этом сумму вероятностей.

Это же верно и для двоек, и для троек, поэтому любое представление можно преобразовать в три группы из идущих подряд одинаковых цифр, не уменышив сумму $p_{1}+p_{2}+p_{3}$. Например, представление на рисунке 5 преобразуется в представление с не меньшей суммой вероятностей на рисунке 7 .

Представлениям из трех групп, идущих в порядке $2 \rightarrow 3 \rightarrow 1,3 \rightarrow 1 \rightarrow 2$ или $1 \rightarrow 2 \rightarrow 3$ (как на рисунке 7 ), соответствует сумма вероятностей 2. Для остальных случаев сумма вероятностей равна 1. Взяв любые три кости, мы можем преобразовать их представление в представление из трех групп с суммой вероятностей не больше двух. Так как при преобразовании сумма вероятностей не уменьшается, для любых трех костей верно неравенство ${ }^{1}$ :

$$
p_{1}+p_{2}+p_{3} \leq 2 \text {. }
$$

Для нетранзитивных костей верна оценка сильнее.

Теорема. Для mрех иестигранных нетранзитивных костей выполняется нера венство

$$
p_{1}+p_{2}+p_{3} \leq \frac{17}{9} .
$$

Для доказательства нам понадобится различать представления по количеству

${ }^{1}$ Для $N$ костей, играющих друг с другом по кругу, сумма вероятностей не превосходит $N-1$.

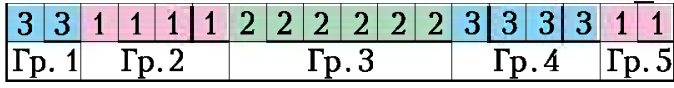

Табл. 2

идущих подряд групп одинаковых чисел. В таблице 2 приведен пример представления, содержащего пять групп.

Назовем представление (и соответствующий набор) оптимальным ${ }^{2}$, если сумму вероятностей нельзя увеличить, поменяв местами два каких-нибудь соседних номера. В оптимальном представлении не встречаются фрагменты $[2 \mid 1],[3 \mid 2]$ и [1|3]. Действительно, если какая-нибудь из этих пар есть, номера в ней можно поменять местами, при этом одна из вероятностей $p_{1}, p_{2}$ и $p_{3}$ увеличится на 1/36.

Любое представление можно сделать оптимальным, меняи местами номера в парах $[2 \mid 1],[3 \mid 2]$ и $[1 \mid 3]$, пока ни одной такой пары не останется. При этом ни одна из вероятностей $p_{1}, p_{2}$ и $p_{3}$ не уменьшится и верно следующее утверждение

Утверждение 1. Для любого набора ко стей с вероятностями $p_{1}, p_{2} u p_{3}$ су иествует оптимальный набор с вероят ностлми $p_{1}^{\prime}, p_{2}^{\prime}$ u $p_{3}^{\prime}$ maкой, ито $p_{1}^{\prime} \geq p_{1}$, $p_{2}^{2} \geq p_{2} u p_{3}^{\prime} \geq p_{3}$

Отсюда следует, что для любого нетранзитивного набора костей найдется оптимальный нетранзитивный набор с не меньшей суммой вероятностей $p_{1}+p_{2}+p_{3}$.

Для доказательства теоремы нам понадобятся несколько вспомогательных утверждений.

Утверждение 2. Для любого оптимального набора из трех костей с иислом групп $n>3$ в представлении найдется опти мальный набор из трех костей с иислом групп $n-1$ и не меньией суммой веромтноcmeü $p_{1}+p_{2}+p_{3}$.

Тем самым, с увеличением числа групп максимально возможная сумма вероятностей оптимального набора костей не возрастает.

Утверждение 3. Максимальнаћ сумма верохтностей для оптимального набора из трех иестигранных костей с количеством групп больие пяти равна 67/36.

Утверждение 4. В представлении любого набора из трех нетранзитивных костей не менее 5 групп.

Термин заимствован из статьи [1]. 


\begin{tabular}{|c|c|c|c|c|c|}
\hline Представление & \begin{tabular}{|l|l|}
1 & 1 \\
\end{tabular} & \begin{tabular}{l|l|l|l|}
2 & 2 & 2 & 2 \\
\end{tabular} & \begin{tabular}{|l|l|l|l|l|l|}
3 & 3 & 3 & 3 & 3 & 3 \\
\end{tabular} & \begin{tabular}{|l|l|l|l|}
1 & 1 & 1 & 1 \\
\end{tabular} & \begin{tabular}{l|l}
2 & 2 \\
\end{tabular} \\
\hline $\begin{array}{l}\text { Номер группы } \\
\text { по порядку }\end{array}$ & 1 & $\begin{array}{llll}1 & 1\end{array}$ & 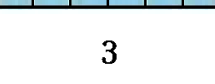 & 4 & 5 \\
\hline $\begin{array}{l}\text { Обозначение } \\
\text { групाы и еe } \\
\text { длина }\end{array}$ & $x_{1}=2$ & $y_{1}=4$ & $z_{1}=6$ & $x_{2}=4$ & $y_{2}=2$ \\
\hline
\end{tabular}

Табл. 3

Утверждение 5. Максимальная сумма вероятностей для трех нетранзитивных иестигранных костей с представлением из пяти групn равна $17 / 9$.

Доказательство теоремы. Возьмем произвольный нетранзитивный набор из трех шестигранных костей. Сделаем, если нужно, его представление оптимальным, меняя местами номера во фрагментах $[2 \mid 1],[3 \mid 2]$ и $[1 \mid 3]$. При этом нетранзитивность сохранится, сумма вероятностей может только увеличиться. В полученном оптимальном нетранзитивном наборе меньше пяти групп быть не может (утверждение 4). Если групп ровно 5, то сумма вероятностей не больше чем 17/9 (утверждение 5). Если групп больше пяти, то сумма вероятностей нового набора не больше чем 67/36 (утверждение 3 ), а это меныше чем $17 / 9$.

Мы взяли произвольный нетранзитивный набор из трех шестигранных костей, преобразовали его, не уменьшив сумму вероятностей, при этом эта сумма оказалась не больше $17 / 9$. Значит, у любых нетранзитивных костей сумма вероятностей не больше 17/9 .

Максимально возможная сумма вероятностей 17/9 достигается для нетранзитивных костей на рисунке 1 . Если при игре в три нетранзитивные шестигранные кости первый игрок выбирает кость случайно, а второй играет наилучшим образом, то вероятность выигрыша второго не превышает

$$
\frac{p_{1}+p_{2}+p_{3}}{3}=\frac{17}{27}
$$

\section{Доказательства утверждений 2-5}

Начиная с этого момента, будем для простоты и определенности считать, что наибольшее число из встречающихся в наборе костей находится на кости номер 1. Тогда все возможные представления начинаются с единицы, а в оптимальных представлениях группы чередуются в порядке $12312312 \ldots$, где цифра 1 означает группу, состойщую из единиц, и т.д.

Доказательство утверждения 2. В оптимальном представлении расставим скобки, заключив в них фрагменты, состоящие из трех последовательных групп (123), и последний фрагмент из одной или двух групп, если он есть. Получается одно из трех разбиений:

$$
\begin{gathered}
(123)(123) \ldots(123), \\
(123)(123) \ldots(123)(12), \\
(123)(123) \ldots(123)(1) .
\end{gathered}
$$

Обозначим через $x_{j}, y_{j}$ и $z_{j}$ первую, вторую и третью группы в $j$-й скобке. Эти обозначения будем применять не только для самих групп, но и для их длин, поскольку путаницы здесь не возникнет. В таблице 3 показан пример. Оптимальное представление можно записать в виде последовательности длин групп $x_{1} y_{1} z_{1} x_{2} y_{2} z_{2} \ldots$ Рассмотрим три случая в зависимости от того, какой группой оканчивается представление.

Случай 1. Представление оканчивается группой $z: x_{1} y_{1} z_{1} x_{2} y_{2} z_{2} \ldots x_{k} y_{k} z_{k}$, общее число групп равно $n k$, где $k \geq 2$

а) Если $x_{1} \leq y_{1}$ то $x_{2}+x_{3}+\ldots+x_{k} \geq$ $\geq y_{2}+y_{3}+\ldots+y_{k}$. Перенесем группу $z_{k}$ влево, объединим ее с группой $z_{1}$ и объединенную группу снова назовем $z_{1}$ :

$$
x_{1} y_{1} z_{1} z_{k} x_{2} y_{2} z_{2} \ldots x_{k} y_{k} \rightarrow{ }_{x_{1} y_{1} z_{1} x_{2} y_{2} \ldots x_{k} y_{k}}
$$

Получится оптимальный набор с не меньшей суммой вероятностей и количеством групп $n-1$. Сумма вероятностей не уменьшается, так как каждая тройка из самой правой группы «перешагивает», двигаясь справа налево, не меньше единиц, чем двоек. Значит, вероятность $p_{3}$ выросла не меньше, чем уменьшилась вероятность $p_{2}$.

б) Если $x_{1}>y_{1}$, то перенесем $z_{k}$ из конца в начало представления:

$$
z_{k} x_{1} y_{1} z_{1} x_{2} y_{2} z_{2} \ldots x_{k} y_{k} \text {. }
$$

Представление остается оптимальным, число групп не изменилось. Сумма вероятностей тоже не изменилась, так как вероятность $p_{3}$ выросла ровно на столько, на сколько уменьшилась вероя̆тность $p_{2}$. Теперь первой оказалась группа троек. 
Циклически переименуем кости (и, стало быть, группы) и снова получим представление $x_{1} y_{1} z_{1} x_{2} y_{2} z_{2} \ldots x_{k} y_{k} z_{k}$.

Будем повторять эту процедуру до тех пор, пока на каком-то шаге не окажется, что $x_{1} \leq y_{1}$, а это обязательно произойдет: невозможно, чтобы было $x_{1}>y_{1}>z_{1}>\ldots$ $\ldots>x_{k+1}>y_{k+1}>z_{k+1}>x_{1}$. Когда выполнится условие $x_{1} \leq y_{1}$, применим процедуру из пункта а) и получим оптимальные кости с количеством групп $n-1$ и суммой вероятностей не меньшей, чем была.

Случай 2. Представление оканчивается группой $x: x_{1} y_{1} z_{1} x_{2} y_{2} z_{2} \ldots x_{k+1}$, общее число групп равно $n=3 k+1$, где $k \geq 1$.

В этом случае между группами $x_{1}$ и $x_{k+1}$ находится поровну двоек и троек, поэтому группу $x_{k+1}$ можно перенести влево и объединить с группой $x_{1}$ без изменения суммы вероятностей:

$\boldsymbol{x}_{1} \boldsymbol{x}_{k+1} y_{1} z_{1} x_{2} y_{2} z_{2} \ldots z_{k} \rightarrow \boldsymbol{x}_{1} y_{1} z_{1} x_{2} y_{2} z_{2} \ldots z_{k}$

После слияния $x_{1}$ и $x_{k+1}$ в одну новую группу $x_{1}$ получается оптимальный набор костей, где сумма вероятностей прежняя, а число групп равно $n-1$.

Случай 3. Представление оканчивается группой $y: x_{1} y_{1} z_{1} x_{2} y_{2} z_{2} \ldots x_{k+1} y_{k+1}$, общее число групп равно $n=3 k+2$, где $k \geq 1$.

Между группами $y_{1}$ и $y_{k+1}$ заключены все группы $z$ и все группы $x$, кроме $x_{1}$. Поэтому единиц между $y_{1}$ и $y_{k+1}$ меньше, чем троек. Перенесем группу $y_{k+1}$ влево и сольем с группой $y_{1}$

$x_{1} y_{1} y_{k+1} z_{1} x_{2} y_{2} z_{2} \ldots x_{k+1} \rightarrow$

$x_{1} y_{1} z_{1} x_{2} y_{2} z_{2} \ldots x_{k+1}$

Получается оптимальный набор из $n-1$ групп, а сумма вероятностей выросла.

Все случаи исчерпаны. Утверждение доказано.

Доказательство утверждения 3. Вначале докажем, что для трех оптимальных шестигранных костей с шестью группами в представлении максимальная сумма вероятностей равна 67/36.

В оптимальном представлении группы чередуются в порядке 123123 . Обозначим длины первых трех групп $x, y$ и $z$. Тогда длины всех шести последовательно расположенных групп равны $x, y, z,(6-x),(6-y)$ и $(6-z)$. Все группы не пустые: $1 \leq x \leq 5,1 \leq y \leq 5$, $1 \leq z \leq 5$, поэтому

$$
\left\{\begin{array}{l}
p_{1}=\frac{6 x+(6-x)(6-y)}{36}=\frac{36-6 y+x y}{36}, \\
p_{2}=\frac{6 y+(6-y)(6-z)}{36}=\frac{36-6 z+x y}{36}, \\
p_{3}=\frac{z(6-x)}{36}=\frac{6 z-z x}{36} .
\end{array}\right.
$$

Сумма вероятностей равна

$$
R=p_{1}+p_{2}+p_{3}=2+\frac{y(z+x-6)-z x}{36}
$$

Рассмотрим три случая в зависимости от знака выражения $z+x-6$.

Слуиай 1: $z+x-6>0$. Тогда $R$ растет с ростом $y$, поэтому максимум будет при $y=5$. Перебором возможных значений $x$ и $z$ найдем наибольшее значение выражения $5 z+5 x-z x-30$ (табл. 4). Поскольку выражение симметрично, можно считать, что $x \leq z$.

Случай 2: $z+x-6=0$. Наибольшее значение выражения $-z x$ равно -5 и достигается, например, при $x=1, z=5$.

Случай 3: $z+x-6<0$. Тогда $R$ убывает с ростом $y$, поэтому наибольшее значение $R$ принимает при $y=1$. Найдем наибольшее значение выражения $z+x-6-z x$ перебором (табл. 5). Снова пользуясь симметрией, ограничимся условием $x \leq z$.

Во всех трех случаях наибольшее значение $R$ равно

$$
2-\frac{5}{36}=\frac{67}{36} \text {. }
$$

В соответствии с утверждением 2 сумма вероятностей оптимальных наборов костей не возрастает с ростом числа групп. Значит, сумма вероятностей выигрыша оптимальных костей с представлением из шести и более групп не превосходит 67/36. Утверждение доказано.

\begin{tabular}{|c|c|c|c|c|c|c|}
\hline$x$ & 2 & 3 & 3 & 4 & 4 & 5 \\
\hline$z$ & 5 & 4 & 5 & 4 & 5 & 5 \\
\hline $5 z+5 x-z x-30$ & -5 & -7 & -5 & -6 & -5 & -5 \\
\hline
\end{tabular}

Табл. 4

\begin{tabular}{|c|c|c|c|c|c|c|}
\hline$x$ & 1 & 1 & 1 & 1 & 2 & 2 \\
\hline$z$ & 1 & 2 & 3 & 4 & 2 & 3 \\
\hline$z+x-6-z x$ & -5 & -5 & -5 & -5 & -6 & -7 \\
\hline
\end{tabular}

Табл. 5 


\section{НАМ ПИШУТ}

\begin{tabular}{|c|c|c|c|c|c|}
\hline \multirow{2}{*}{ Число граней } & \multicolumn{5}{|c|}{ Длина группы } \\
\cline { 2 - 6 } & Группа 1 & Группа 2 & Группа 3 & Группа 4 & Группа 5 \\
\hline$N \geq 6, N$ четное & 2 & $\frac{N}{2}+1$ & $N$ & $N-2$ & $\frac{N}{2}-1$ \\
\hline$N \geq 3, N$ нечетное & 1 & $\frac{N+1}{2}$ & $N$ & $N-1$ & $\frac{N-1}{2}$ \\
\hline
\end{tabular}

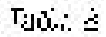

Для $N=4$ нетранзитивных костей с представлением из пяти групп не суиествует.

Доказываются эти обобщенные утверждения аналогично случаю $N=6$, только возрастает техническая сложность при нахождении максимумов выражений. Читатель может попробовать сделать соответствующие расчеты самостоятельно.

Теперь теорему о максимальной сумме вероятностей для трех нетранзитивных костей можно сформулировать так:

Теорема для $\boldsymbol{N}$-гранных костей. Для трех нетранзитивных костей с $N$ гранями каждая выполняются неравенства

$p_{1}+p_{2}+p_{3} \leq 2-\frac{N-2}{\lambda^{2}}$ для четных $N \geq 6$;

$$
p_{1}+p_{2}+p_{3} \leq 2-\frac{N-1}{2 N^{2}}
$$

для нечетных $N \geq 3$.

Максимальная сумма вероятностей достигается для оптимальных наборов костей с представлением из пяти групп и длинами групп, показанными в таблице 8 .

\section{Литература}

1. И.И.Богданов. Нетранзитивные рулетки. Математическое просвещение, сер. 3, вып. 14, c. $240-255$.

2. М.Гарднер. Нетранзитивные парадоксы. В книге «Путешествие во времени». - М.: Мир, 1990.

\section{НАМ ПИІІТ}

Недавно в редакцию нашего журнала пришло такое письмо.

Уважаемые квантовцы!

Рискну предложить вам Метрический кроссворд - эдакую словесную игру-загадку, в которой все зашифрованные термины имеют общий корень (или один из корней) «метр».

Отмечу, что именно под влиянием «Кванта» в далекие 1970-е я, страстный поклонник гуманитарных наук, отдал предпочтение техническому вузу - Киевскому политехническому институту (выбрав специальность, где господствовали термометры и калориметры).

Свою непродолжительную практическую инженерно-техническую деятельность связал с отраслью, где властвовали манометры и дозиметры- на монтаже 4-го и 5-го энергоблоков ЧАЭС и ликвидации последствий Чернобыльской аварии.

После әтого приобщился к содружеству профессиональных почитателей миллиметровки - в проектном институте «Киевский промстройпроект». Затем на два дестилетия с головой ушел в сферу, где в большой чести господствовали одиннадцатиметровый и стометровка, - в спортивную журналистику.

И вот уже полвека храню верность кроссвордистике. Шутка сказать - мой первый кроссворд в «Кванте» был напечатан еще в 1979 году.

С уважением, В.Закревский

Этот кроссворд мы публикуем на 4-й странице обложки нашего журнала. 